\title{
Emergence of Novel Pathogenic Streptomyces Species by Site-Specific Accretion and cis-Mobilization of Pathogenicity Islands
}

\author{
Yucheng Zhang and Rosemary Loria \\ Department of Plant Pathology, University of Florida, Gainesville, U.S.A.
}

Accepted 15 December 2016.

The main pathogenicity factor of Streptomyces species associated with the potato common scab disease is a nitrated diketopiperazine called thaxtomin A (ThxA). In Streptomyces scabiei (syn. S. scabies), which is thought to be the most ancient pathogenic Streptomyces species, the ThxA biosynthetic cluster is located within a mobile genomic island called the toxicogenic region (TR). Three attachment (att) sites further separate TR into two subregions (TR1 and TR2). TR1 contains the ThxA biosynthetic cluster and is conserved among several pathogenic Streptomyces species. However, TR2, an integrative and conjugative element, is missing in most pathogenic species. In our previous study, we demonstrated the mobilization of the whole TR element or TR2 alone between $S$. scabiei and nonpathogenic Streptomyces species. TR1 alone did not mobilize in these experiments. These data suggest that TR2 is required for the mobilization of TR1. Here, we show that TR2 can self mobilize to pathogenic Streptomyces species harboring only TR1 and integrate into the att site of TR1, leading to the tandem accretion of resident TR1 and incoming TR2. The incoming TR2 can further mobilize resident TR1 in cis and transfer to a new recipient cell. Our study demonstrated that TR1 is a nonautonomous cis-mobilizable element and that it can hijack TR2 recombination and conjugation machinery to excise, transfer, and integrate, leading to the dissemination of pathogenicity genes and emergence of novel pathogenic species. Additionally, comparative genomic analysis of 23 pathogenic Streptomyces isolates from ten species revealed that the composite pathogenicity island (PAI) formed by TR1 and TR2 is dynamic and various compositions of the island exist within the population of newly emerged pathogenic species, indicating the structural instability of this composite PAI.

Although Streptomyces spp. are viewed predominantly as free-living terrestrial soil-inhabiting bacteria, some species are symbionts with fungi, insects, plants, and animals (Seipke et al. 2012) and some strains reside in marine soils (Fiedler et al. 2005). A few Streptomyces species are plant pathogens and cause diseases of underground crop structures, such as roots and tubers; the most economically important disease caused by Streptomyces species is potato scab, characterized by raised, pitted, or superficial lesions formed on the tuber surface (Loria et al. 2006). The

Corresponding author: R. Loria; E-mail: rloria@ufl.edu

*The $\boldsymbol{e}$-Xtra logo stands for "electronic extra" and indicates that three supplementary figures and one supplementary table are published online.

(c) 2017 The American Phytopathological Society scab-causing species Streptomyces scabiei (syn. S. scabies) also infects the pericarp of the peanut fruit, causing peanut pod wart disease; however, this little-known disease has only been described in Israel (Kritzman et al. 1996) and South Africa (de Klerk et al. 1997).

Though $S$. scabiei is thought to be the most ancient scab-causing Streptomyces species (Bukhalid et al. 2002), there are a number of other species that are also the causal agents of scab lesions on actively growing tubers. The best-characterized scab-causing species are S. scabiei, S. acidiscabies, and S. turgidiscabies (Labeda 2016). An additional pathogenic species, $S$. ipomoeae, incites the soil rot disease on sweet potato (Ipomoea batatas [L.] Lam.) (Tomihama et al. 2016). Besides these four well-studied pathogenic species, seven other potato scab-causing Streptomyces species have also been described, namely S. europaeiscabiei, S. stelliscabiei, S. luridiscabiei, S. puniciscabiei, S. niveiscabiei, S. reticuliscabiei, and S. caviscabies (Bouchek-Mechiche et al. 2000; Goyer et al. 1996; Park et al. 2003a).

The primary pathogenicity determinant of scab-causing Streptomyces species is a family of dipeptide phytotoxins known as thaxtomins (Loria et al. 2006). A total of 11 family members have been isolated; these thaxtomin analogs are differentiated by the presence or absence of either hydroxyl, Nmethyl groups, or both (Bignell et al. 2010a). In S. scabiei, $S$. acidiscabies, and $S$. turgidiscabies, thaxtomin A (ThxA) is the predominant family member (Healy et al. 2000; King et al. 1989; Lawrence et al. 1990). However, the major thaxtomin analog produced by $S$. ipomoeae is the nonhydroxylated analog called thaxtomin C (King et al. 1994). S. europaeiscabiei, S. stelliscabiei, S. niveiscabiei, and S. caviscabies also produce thaxtomins (Goyer et al. 1996; Loria et al. 2006; Park et al. 2003a). However, thaxtomin production by $S$. luridiscabiei and S. puniciscabiei, two species only reported in Korea (Park et al. 2003a), has not been demonstrated (Park et al. 2003b).

In $S$. turgidiscabies Car8, ThxA biosynthetic genes and other virulence genes, including necl, a tomatinase gene (tomA), and a plant fasciation (fas) operon, reside on a mobile 674-kb pathogenicity island (PAI) (Huguet-Tapia et al. 2011; Kers et al. 2005). In contrast, virulence factors in $S$. scabiei and $S$. acidiscabies reside on two remote chromosomal regions, namely the toxicogenic region (TR) and the colonization region (CR) (Lerat et al. 2009). CR contains necl and tomA (Lerat et al. 2009). In S. scabiei, TR is further separated into two subregions (TR1 and TR2) by an internal and two flanking attachment (att) sites (Chapleau et al. 2016). TR1 harbors the ThxA biosynthetic gene cluster and TR2 possesses a putative integrative and conjugative element (ICE) (Chapleau et al. 2016). ICEs are self-transmissible elements that encode the machinery for excision, conjugation, and integration as well as regulatory systems to regulate their excision and 
conjugative transfer (Burrus and Waldor 2004; Osborn and Böltner 2002; Salyers et al. 1995). Chapleau et al. (2016) demonstrated the excision of TR from S. scabiei in three distinct forms: as TR1 alone, as TR2 alone, and as the entire TR element; reintegration of the TR elements was not reported. Recently, we demonstrated the site-specific integration of TR2 alone or the entire TR from S. scabiei into the chromosome of the soil saprophyte Streptomyces diastatochromogenes ATCC 12309; acquisition of the entire TR but not TR2 alone conferred a pathogenic phenotype on $S$. diastatochromogenes (Zhang et al. 2016). In this study, independent integration by TR1 was not observed, suggesting that TR1 may be a cis-mobilizable element (CIME). CIMEs are genomic islands that lack proteins involved in recombination and conjugation but are flanked by att sites (Bellanger et al. 2014). We also observed that TR2, which is a putative ICE, only exists in S. scabiei and a novel pathogenic species (Streptomyces sp. strain 96-12, GenBank accession number GCA_001550315.1) but is absent in other pathogenic species, such as $S$. acidiscabies and $S$. europaeiscabiei (Zhang et al. 2016). These data suggest that S. scabiei is likely to be the source of the entire TR region and that its mobilization contributes to the emergence of novel pathogenic species (Zhang et al. 2016).

Our previous study found that $S$. acidiscabies 84-104, an emergent pathogen (Huguet-Tapia and Loria 2012), has a TR1 region that is almost identical to the TR1 region in $S$. scabiei; however, TR2 is absent from S. acidiscabies 84-104 (Zhang et al. 2016). We hypothesized that $S$. acidiscabies first acquired the entire TR element but, subsequently, lost TR2. In the current study, we sequenced the genomes of an additional four S. acidiscabies isolates from different locations to explore the diversity of TR elements within the population of S. acidiscabies. This study benefited from the very recent availability of genome sequences for plant-pathogenic streptomycetes (Huguet-Tapia et al. 2016; Labeda 2016; Tomihama et al. 2016). These genomes allowed us to characterize the variation in the sequences of PAIs within and among pathogenic Streptomyces species and to investigate the origin and evolution of pathogenicity in the Streptomyces genus. Here, we demonstrate that TR2 can transfer itself to a pathogenic isolate harboring only TR1 and integrate into one of the two att sites flanking TR1, leading to the tandem accretion of resident TR1 and incoming TR2. Furthermore, the resulting composite genomic island can excise and be transferred to a new host. These data demonstrated that TR1 is a CIME and the site-specific accretion and cis-mobilization of TR1 together with the related ICE (TR2) have a key role in the dissemination of pathogenicity and virulence genes and emergence of novel pathogenic species in Streptomyces genus.

\section{RESULTS}

\section{Pathogenicity and ThxA production \\ of four $S$. acidiscabies isolates.}

Two $S$. acidiscabies isolates from the United States (85-06, FL01) and two isolates from Japan (98-48, 98-49) were selected for genome sequencing (Table 1). Three of these four $S$. acidiscabies isolates and three well-studied pathogenic isolates (S. acidiscabies 84-104, S. turgidiscabies Car8, and S. scabiei 87-22) caused the expected disease symptoms on radish seedlings (Fig. 1A). Noninoculated seedlings and seedlings inoculated with the nonpathogenic $S$. diastatochromogenes grew normally. In contrast, S. acidiscabies $98-48$ had a phenotype intermediate to the healthy seedlings and those inoculated with the other pathogenic strains.

We compared ThxA production by the four $S$. acidiscabies strains (85-06, FL01, 98-48, and 98-49) with three well-studied pathogenic isolates, namely, S. acidiscabies 84-104, S. scabiei 87-22, and S. turgidiscabies Car8 in oat bran broth (OBB) (Fig. 1B), a complex medium used to induce ThxA biosynthesis (Wach et al. 2007). Four of the five $S$. acidiscabies isolates (84-104, 8506, 98-49, and FL01), S. turgidiscabies Car8, and S. scabiei 87-22 produced ThxA as expected. However, S. acidiscabies 98-48 did not produce a detectable level of ThxA in OBB, which can explain why this strain cannot induce the expected pathogenic phenotype on radish seedlings (Fig. 1A). We also found that all of the four ThxA-producing $S$. acidiscabies isolates (84-104, 85-06, 98-49, and FL01) produced a significantly greater quantity of ThxA than S. scabiei.

\section{Phylogenomic and genome similarity analysis of pathogenic and related nonpathogenic species.}

Previously, we demonstrated the power of core genomebased phylogenetic analysis to infer evolutionary relationships

Table 1. Genome summary statistics of plant-pathogenic Streptomyces spp.

\begin{tabular}{|c|c|c|c|c|c|c|c|c|}
\hline Species & Strain name & $\begin{array}{l}\text { Genome } \\
\text { status }\end{array}$ & Contig no. & $\begin{array}{c}\text { Contig } \\
\text { N50 }(\text { kb) }\end{array}$ & $\begin{array}{c}\text { Genome } \\
\text { size }(\mathrm{Mb})\end{array}$ & Geographic location & $\begin{array}{c}\text { GenBank assembly } \\
\text { accession no. }\end{array}$ & Source or reference \\
\hline S. acidiscabies & 84-104 & Draft & 244 & 113.4 & 11.0 & U.S.A.: upstate New York & GCA_000242715.2 & Huguet-Tapia and Loria 2012 \\
\hline S. acidiscabies & $85-06$ & Draft & 1073 & 20.0 & 11.0 & U.S.A.: West Virginia & LYDU00000000 & This study \\
\hline S. acidiscabies & $98-48$ & Draft & 983 & 20.3 & 10.6 & Japan: Saga & LYDS00000000 & This study \\
\hline S. acidiscabies & $98-49$ & Draft & 1211 & 16.5 & 10.2 & Japan: Saga & LYDT00000000 & This study \\
\hline S. acidiscabies & FL01 & Draft & 993 & 19.3 & 10.6 & U.S.A.: Florida & LYDV00000000 & This study \\
\hline S. acidiscabies & $\mathrm{a} 10$ & Draft & 279 & 82.2 & 10.7 & $\begin{array}{l}\text { Japan: Kagoshima, } \\
\text { Nagashima }\end{array}$ & GCA_001485105.1 & Tomihama et al. 2016 \\
\hline S. acidiscabies & NCPPB 4445 & Draft & 263 & 88.0 & 10.0 & Unknown & GCA_001189015.1 & University of Exeter \\
\hline S. europaeiscabiei & NRRL B-24443 & Draft & 1056 & 16.4 & 10.0 & France: Le Rhue & GCA_000988945.1 & Labeda 2016 \\
\hline S. europaeiscabiei & NCPPB 4086 & Draft & 295 & 97.2 & 10.5 & Canada: Ontario & GCA_000738695.1 & University of Exeter \\
\hline S. stelliscabiei & NRRL B-24447 & Draft & 991 & 19.0 & 10.0 & France & GCA_001008135.1 & USDA ARS NCAUR $^{\mathrm{a}}$ \\
\hline S. stelliscabiei & P3825 & Draft & 705 & 32.8 & 10.4 & Unknown & GCA_001189035.1 & University of Exeter \\
\hline S. turgidiscabies & Car8 & Draft & 692 & 27.4 & 10.8 & Japan & GCA_000331005.1 & Huguet-Tapia et al. 2011 \\
\hline S. turgidiscabies & $\mathrm{T} 45$ & Draft & 101 & 264.4 & 10.6 & $\begin{array}{l}\text { Japan: Kagoshima, } \\
\text { Kushira }\end{array}$ & GCA_001485145.1 & Tomihama et al. 2016 \\
\hline S. ipomoeae & $91-03$ & Draft & 687 & 26.4 & 10.4 & U.S.A. & GCA_000317595.1 & Huguet-Tapia et al. 2016 \\
\hline S. scabiei & $87-22$ & Complete & & & 10.1 & U.S.A.: Wisconsin & GCA_000091305.1 & Bignell et al. 2010a and b \\
\hline S. scabiei & NRRL B-16523 & Draft & 1091 & 15.1 & 9.8 & U.S.A.: central New York & GCA_001005405.1 & Labeda 2016 \\
\hline S. scabiei & S58 & Draft & 149 & 193.2 & 10.0 & $\begin{array}{l}\text { Japan: Kagoshima, } \\
\text { Nagashima }\end{array}$ & GCA_001485125.1 & Tomihama et al. 2016 \\
\hline S. luridiscabiei & NRRL B-24455 & Draft & 821 & 18.0 & 7.9 & South Korea: Jeju & GCA_001418625.1 & Labeda 2016 \\
\hline S. puniciscabiei & NRRL B-24456 & Draft & 688 & 25.4 & 8.8 & South Korea: Jeju & GCA_001419685.1 & Labeda 2016 \\
\hline S. niveiscabiei & NRRL B-24457 & Draft & 1568 & 10.1 & 9.5 & South Korea: Jeju & GCA_001419795.1 & Labeda 2016 \\
\hline S. niveiscabiei & ST1015 & Draft & 1126 & 14.6 & 9.8 & Uruguay & LXWC00000000 & Universidad de la República \\
\hline S. niveiscabiei & ST1020 & Draft & 1010 & 17.8 & 9.9 & Uruguay & LXWD00000000 & Universidad de la República \\
\hline Streptomyces sp. & $96-12$ & Draft & 1875 & 8.7 & 10.1 & Egypt & GCA_001550315.1 & Zhang et al. 2016 \\
\hline
\end{tabular}

${ }^{a}$ USDA-ARS-NCAUR = United States Department of Agriculture-Agricultural Research Service-National Center for Agricultural Utilization Research. 
among Streptomyces species, and the power of in silico DNADNA hybridization (isDDH) and the average nucleotide identity (ANI) to identify novel pathogenic species (Zhang et al. 2016). However, our previous study did not include less-studied pathogenic Streptomyces species, namely $S$. luridiscabiei, $S$. puniciscabiei, and S. niveiscabiei, whose genomes have been sequenced recently (Labeda 2016). To obtain a better understanding of evolutionary relationships of pathogenic Streptomyces species, we included these less-studied pathogenic species into the phylogenomic and systematic analysis.

A core genome-based maximum likelihood phylogeny of selected pathogenic isolates was constructed by 1,104 single-copy ortholog groups that are shared among all tested pathogenic Streptomyces isolates as well as isolates from their closely related nonpathogenic Streptomyces species (S. bottropensis and S. galbus) and two outgroups (Fig. 2). The phylogenetic tree was distributed into two major clades. The first clade contains S. scabiei, S. stelliscabiei, S. bottropensis ATCC 25435, S. europaeiscabiei, Streptomyces sp. strain 96-12, S. galbus KCCM 41354, S. ipomoeae, and $S$. turgidiscabies; the second clade includes $S$. acidiscabies, S. niveiscabiei, and S. puniciscabiei. S. luridiscabiei, however, cannot be placed into either of these two groups. Although S. bottropensis ATCC 25435 and S. galbus KCCM 41354 are nonpathogenic species, they are more closely related to $S$. scabiei, S. stelliscabiei and S. europaeiscabiei than S. acidiscabies and $S$. niveiscabiei. This indicates that these pathogenic species acquired pathogenicity genes after their ancestor diverged into different species. However, an alternative hypothesis is that S. bottropensis and $S$. galbus lost their pathogenicity determinants after divergence.

ANI and isDDH are two of the most widely accepted in silico tools used to determine strain relationships, and 95 to $96 \%$ ANI and $70 \%$ isDDH values were applied as the threshold for species delineation (Auch et al. 2010a; Goris et al. 2007). In our analysis, we found that isolates in the same phylogenetic clade also showed high ANI and isDDH values (Supplementary Fig. S1). Although $S$. niveiscabiei and $S$. acidiscabies are closely related in the phylogenetic tree (Fig. 2), the low ANI (approximately 93\%) and isDDH (52 to 54\%) values unambiguously indicate that the current classification of these strains into two species is appropriate.

\section{Acquisition of the ThxA biosynthetic genes}

through recent horizontal gene transfer (HGT) events.

To infer the evolution history of ThxA biosynthetic genes, we constructed a phylogenetic tree of pathogenic isolates, using

A

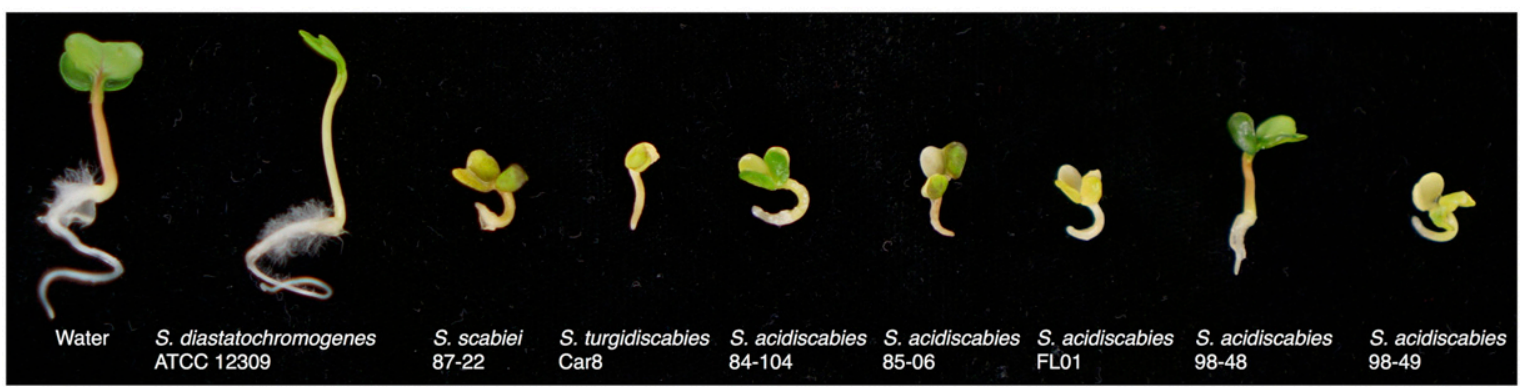

B

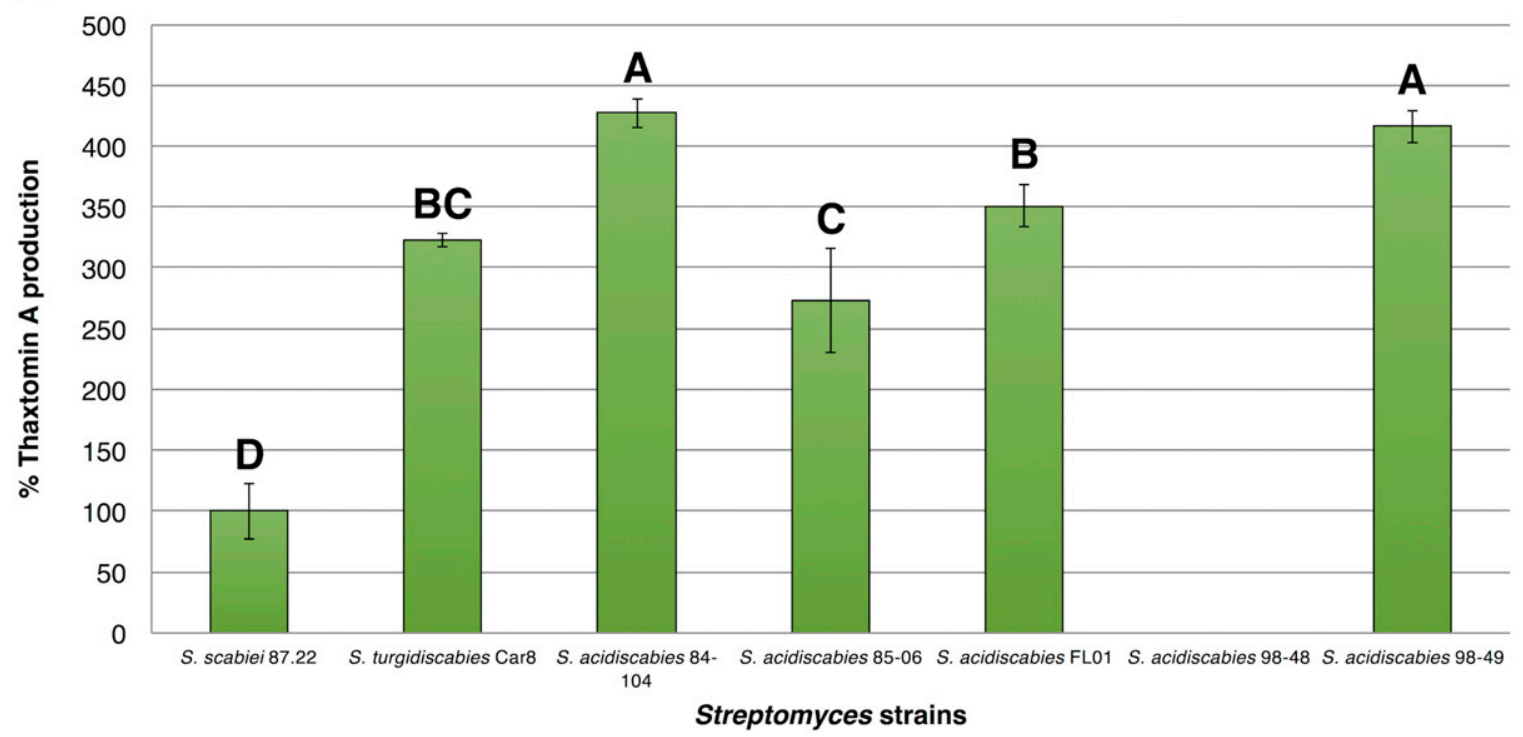

Fig. 1. Virulence assays and thaxtomin A (ThxA) production of pathogenic Streptomyces isolates. A, Surface-sterilized radish seedlings (six per treatment) were inoculated with mycelia from tryptic soy broth-grown cultures of Streptomyces strains. Seedlings treated with S. scabiei 87-22, the nonpathogen S. diastatochromogenes ATCC 12309, or with water were used as controls. Plants were incubated at room temperature under a 12-h photoperiod for 4 days. Representative plants for each treatment are shown. B, Production of ThxA by plant-pathogenic Streptomyces isolates. Isolates were grown in triplicate in oat bran broth medium for 6 days at $28^{\circ} \mathrm{C}$. The average thaxtomin production of S. scabiei $87-22$ that is normalized using the dried cell weight is set to $100 \%$. The average percent production for each strain relative to S. scabiei 87-22 and normalized using dried cell weights is shown. The error bars represent the standard deviation from the mean. Letters represent results of a one-way analysis of variance with Tukey's honestly significantly different (HSD) test; bars not sharing letters are significantly different at $P<0.05$. 
their $t x t A, t x t B, t x t C, t x t D, t x t E, t x t H$, and $t x t R$ genes (Supplementary Fig. S2). In the ThxA tree, five $S$. acidiscabies isolates (a10, 84-104, 85-06, 98-49, and FL01), two S. scabiei isolates (87-22 and NRRL B-16523), S. niveiscabiei NRRL B-24457, and Streptomyces sp. strain 96-12 were closely grouped, indicating the high similarity of their ThxA clusters. In contrast, S. acidiscabies, S. niveiscabiei, Streptomyces sp. strain 96-12, and $S$. scabiei were distantly related in the core-genome tree (Fig. 2). The incongruence (Fig. 3) between the core-genome phylogeny (Fig. 2) and the ThxA phylogeny supports the notion that a recent HGT of ThxA clusters occurred in these strains.

\section{TR1 can excise from the chromosome} of pathogenic Streptomyces strains lacking TR2.

The excision of TR regions from the chromosome of S. scabiei $87-22$ was detected by nested polymerase chain reaction (PCR) assays. However, in strains lacking TR2, the TR1 element did not excise (Chapleau et al. 2016). In our analysis, we found that the regions flanking TR elements used for primer design in the study by Chapleau et al. (2016) were not conserved in different pathogenic species. We hypothesized that this is the reason why they did not detect the excision of TR1 elements in strains lacking TR2. To re-analyze excision of TR1, we designed nested PCR primers to detect the circular form of TR1 and the corresponding repaired chromosomal junctions formed upon excision in S. acidiscabies 84-104, which contains only TR1. Excision of TR1 was observed in S. acidiscabies 84-104 (Fig. 4A and B), indicating TR1 can excise in strains lacking the TR2 region. Surprisingly, the sequence of the PCR products showed that the excision of TR1 in S. acidiscabies 84-104 did not occur at the previously identified att sites (TTGAAGCGGAAC) (Chapleau et al. 2016; Zhang et al. 2016) but via a different repeated sequence (TTTGCCAGATCAG) (Fig. 4C). This result demonstrates that the excision mechanism of S. acidiscabies 84104 TR1 may be different from that of the $S$. scabiei TR region.

\section{PAI variation exists}

within pathogenic Streptomyces species.

Understanding the evolution of Streptomyces PAIs is important to our understanding of the emergence of pathogenic

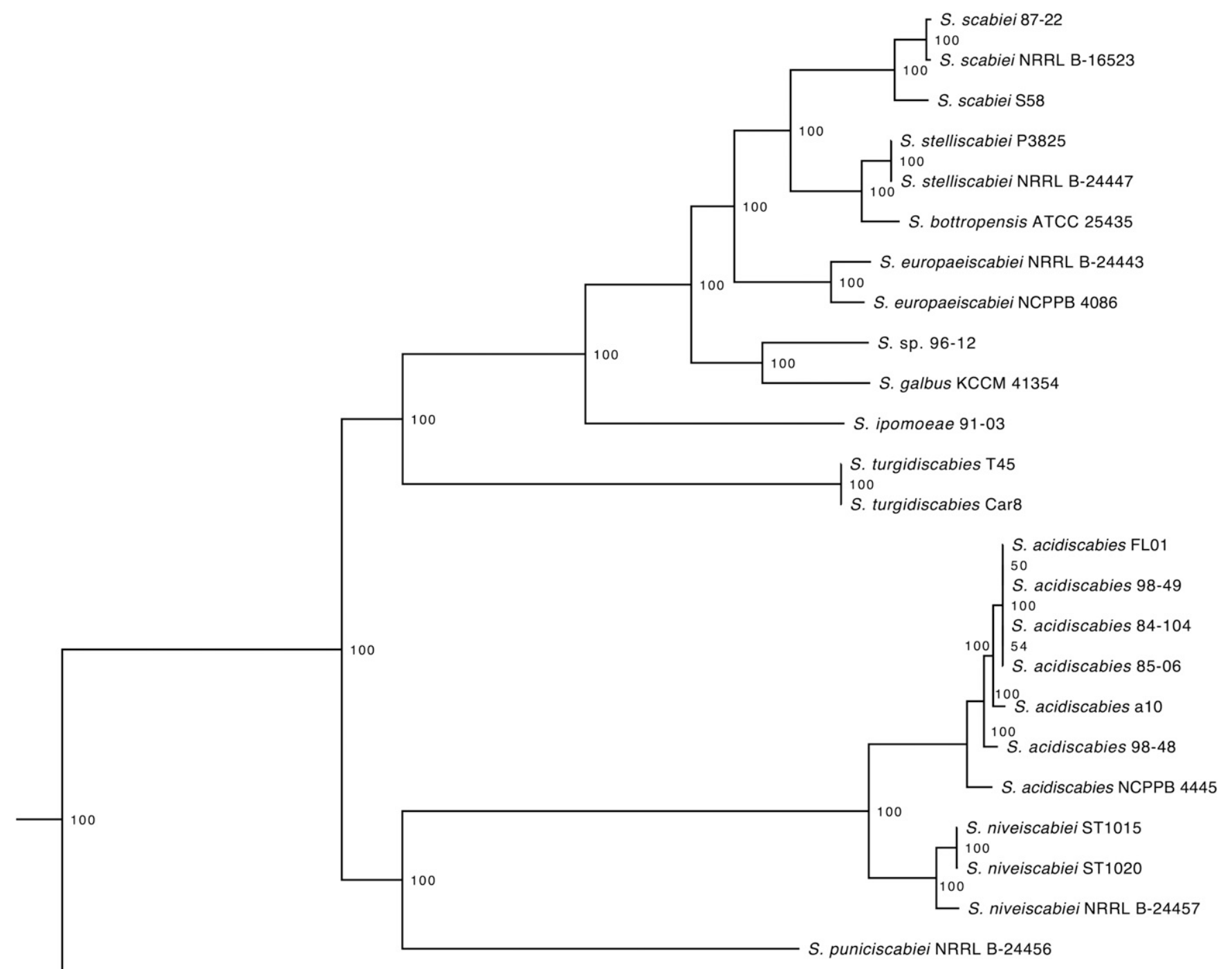

S. Iuridiscabiei NRRL B-24455

0.03

Fig. 2. Phylogeny of pathogenic Streptomyces species and their related nonpathogenic Streptomyces species, based on the concatenation of 1,104 single-copy gene clusters with the maximum likelihood algorithm RAxML. 
Streptomyces species in agricultural systems. The best strategy we have to approach this goal is to identify and compare the PAIs from pathogens utilizing whole genome sequences (Zhang et al. 2016). In our previous study, we have shown that TR is the most common PAI in scab-causing Streptomyces species, except in S. turgidiscabies Car8 (Zhang et al. 2016). Here, we expand on previous analyses and include newly available genome sequences, including those reported here (Fig. 5).

We had previously reported that TR2, which is an ICE, only existed in S. scabiei and Streptomyces sp. strain 96-12 but not in other pathogenic species, such as $S$. acidiscabies and S. europaeiscabiei (Zhang et al. 2016). In this study, we identified the complete TR element (TR1 and TR2) in the newly sequenced genome of $S$. acidiscabies a10 (Tomihama et al. 2016). However, $S$. acidiscabies $98-48$ and the newly released sequence of $S$. acidiscabies NCPPB 4445 lack both TR1 and TR2; this is consistent with the lack of pathogenicity and thaxtomin production by $S$. acidiscabies $98-48$ (Fig. 1). Therefore, within the $S$. acidiscabies species, three distinct compositions of the TR element exist: i) TR1 alone, ii) the entire composite TR (TR1+TR2), and iii) neither TR1 nor TR2 (Fig. 5). The genomes of $S$. niveiscabiei isolates also vary in their TR composition. S. niveiscabiei NRRL B-24457, isolated from South Korea, contains TR1, while S. niveiscabiei ST1015 and S. niveiscabiei ST1020, two strains recently isolated from pitted potato scab lesions in Uruguay (M. Julia-Pianzzola personal communication), do not contain either TR1 or TR2.

Analysis of the genomes of two S. stelliscabiei isolates (NRRL B-24447 and P3825) suggests that these two isolates contain both TR1 and TR2. In these S. stelliscabiei assemblies, the TR1 regions were complete but the TR2 regions were separated into several contigs. For example, in S. stelliscabiei P3825, TR1 and part of TR2 reside on a 111.4-kb contig (Genbank accession number JPPZ01000626.1), and the other part of TR2 resides on a 102.4-kb contig (Genbank accession number JPPZ01000624.1). Similar to $S$. scabiei, the TR2 region of S. stelliscabiei $\mathrm{P} 3825$ was also inserted into the att site at the $3^{\prime}$ end of aviX1 gene. Chapleau et al. (2016) described the genes in TR2 that may be involved in conjugation and integration. S. stelliscabiei P3825 possesses all of these genes, except the gene encoding the ATPase (SCAB_32961). Whether the lack of this ATPase gene affects the mobilization of
S. stelliscabiei TR requires further investigation. In addition, comparison of the TR regions in S. scabiei and S. stelliscabiei revealed significant genetic variation (data not shown), suggesting that the TR regions of S. stelliscabiei and S. scabiei have evolved independently for some time.

$S$. turgidiscabies Car8 is an emergent pathogen first reported in Japan (Miyajima et al. 1998; Takeuchi et al. 1996). S. turgidiscabies Car8 does not contain the TR region but it contains a large mobile PAI of $674 \mathrm{~kb}$ (Huguet-Tapia et al. 2011; Kers et al. 2005). Recently, the genome of another Japanese isolate, S. turgidiscabies S58, was sequenced (Tomihama et al. 2016). Although the $S$. turgidiscabies S58 assembly is not complete, we identified several contigs of the S. turgidiscabies S58 assembly that share synteny with the 674-kb PAI of $S$. turgidiscabies Car8. These data indicate that the two $S$. turgidiscabies isolates appear to contain the same 674-kb PAI, which is
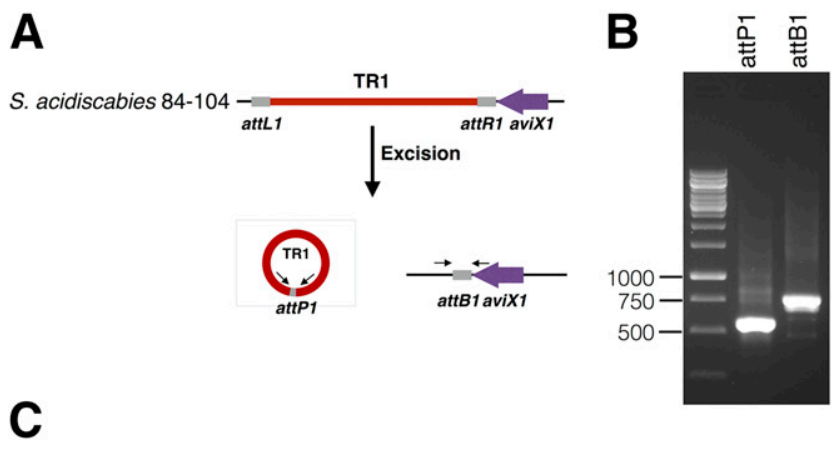

Fig. 4. Excision of the toxicogentic region (TR) in Streptomyces acidiscabies 84-104. A, Illustration of the excision of TR1 from the chromosome of S. acidiscabies 84-104. Arrows represent primers used to amplify the attachment site (attPl) formed upon the excision of a circular module of TR1 and repaired chromosomal junction (attB1). B, Nested polymerase chain reaction products of $a t t P 1$ and $a t t B 1$. C, TR1 is excised from the chromosome of $S$. acidiscabies $84-104$ by site-specific recombination between two 13-bp direct repeats (attL1 and attRl).



Fig. 3. Comparison of evolutionary relationships of phytopathogenic Streptomyces species derived from the core-genome tree (left) and the thaxtomin A (ThxA) tree (right). 
consistent with the previous conception of a high homologous PAI in S. turgidiscabies isolates from Hokkaido Island, Japan (Bukhalid et al. 1998; Miyajima et al. 1998).

\section{Site-specific accretion}

between resident TR1 and incoming TR2.

Recently, we demonstrated that TR2 had a high integration specificity, as it was always integrated into the att site at the $3^{\prime}$ end of aviX1, which encodes a putative ATP/GTP binding protein (Zhang et al. 2016). Since $S$. scabiei TR1 is not mobile by itself (Zhang et al. 2016), we hypothesized that cis-mobilization of Streptomyces TR1-TR2 is via site-specific accretion. To test this hypothesis, we used strains from previous studies in mating experiments: i) S. acidiscabies 84. 303. 1 (kanamycin resistance $\left[\mathrm{kan}^{\mathrm{R}}\right]$ ) (Healy et al. 2000), containing only TR1 with a deletion mutation in $t x t A$ and $t x t B$ genes (hereinafter referred to as $S$. acidiscabies $\triangle t x t A B)$, and ii) the $S$. diastatochromogenes transconjugant strain (hygromycin B resistance $\left[\mathrm{hyg}^{\mathrm{R}}\right]$ ) that acquired TR2 from S. scabiei with a deletion mutation in lantibiotic biosynthesis genes lanA (SCAB_32021) and lanB (SCAB_32031) (hereinafter referred to as $S$. diastatochromogenes $\triangle$ lan $A B$ ) (Zhang et al. 2016). S. diastatochromogenes $\triangle \operatorname{lan} A B\left(\right.$ hyg $\left.^{\mathrm{R}}\right)$ served as the putative TR2 donor, while $S$. acidiscabies $\triangle \operatorname{txt} A B\left(\operatorname{kan}^{\mathrm{R}}\right)$ with the resident TR1 acted as the putative recipient strain (Figs. 6A and 7). Following selection with kanamycin and hygromycin B, we obtained $S$. acidiscabies transconjugants containing both TR1 and TR2 (hereinafter referred to as $S$. acidiscabies $\triangle \operatorname{txt} A B \triangle$ lan $A B$ ). Using PCR primers $\mathrm{a}+\mathrm{b}, \mathrm{c}+\mathrm{d}, \mathrm{e}+\mathrm{f}$, and $\mathrm{g}+\mathrm{h}$ (Supplementary Table S1; Supplementary Fig. S3), we found that TR2 was always inserted into the TR1 attachment site $(a t t R)$ located at the $3^{\prime}$ end of the $S$. acidiscabies aviX1 gene rather than the attachment site (attL) on the other side of TR1 (Fig. 7). Furthermore, in all 15 $S$. acidiscabies $\triangle \operatorname{txt} A B \triangle \mathrm{Ian} A B$ transconguants tested, the orientation and structure of TR1 and TR2 is the same as TR1 and TR2 in S. scabiei (Fig. 5). These data indicate that, although the resident TR1 has two attachment sites, the incoming TR2 preferably integrates into one attachment site (attR) (Fig. 7), leading to the tandem accretion of two elements.

\section{Conjugative mobilization}

of resident TR1 in cis by incoming TR2.

We then investigated the conjugative transfer of the composite element of resident (S. acidiscabiei) TR1-incoming (S. scabiei) TR2 (Fig. 6B). S. acidiscabies $\triangle \operatorname{txt} A B \quad \Delta \operatorname{lan} A B$ served as the putative TR donor, while the $S$. diastatochromogenes transconjugant (thiostrepton resistance) from a previous study (Kers et al. 2005), containing the 674-kb PAI of S. turgidiscabies Car8 with a deletion mutation in necl (hereinafter referred to as $S$. diastatochromogenes $\Delta n e c l$ ) acted as the putative recipient strain. Following selection with hygromycin B and thiostrepton,

S. scabiei

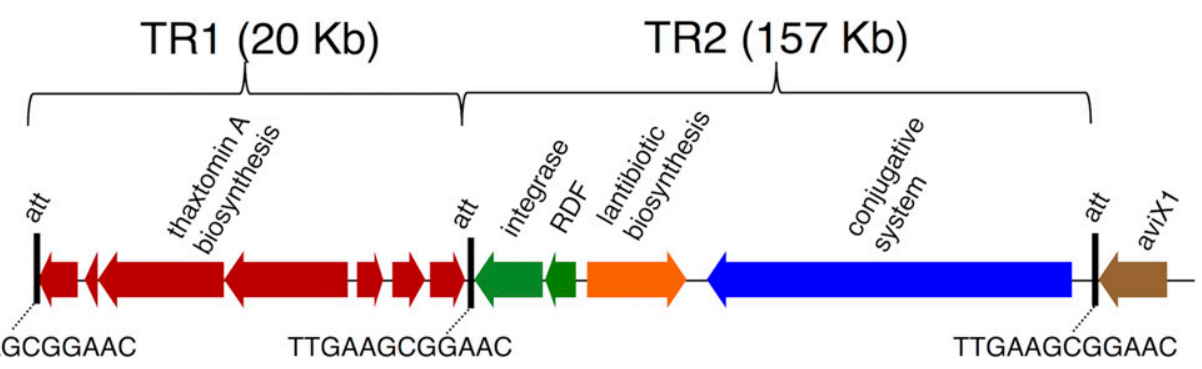

S. acidiscabies $84-104$

S. acidiscabies 85-06

S. acidiscabies FL01

S. acidiscabies $98-49$

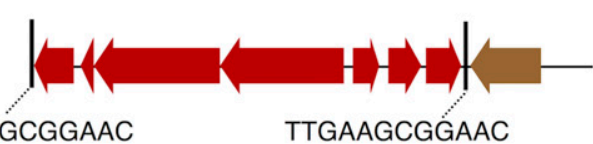

S. acidiscabies $98-48$

S. acidiscabies NCPPB 4445

S. puniciscabiei NRRL B-24456



S. Iuridiscabiei NRRL B-24455

S. niveiscabiei ST1015/ST1020

S. acidiscabies a10

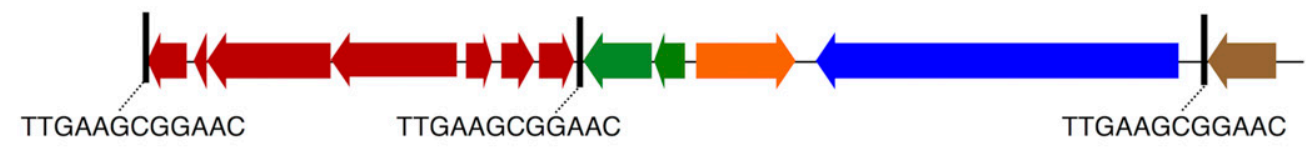

S. niveiscabiei NRRL B-24457

S. stelliscabies

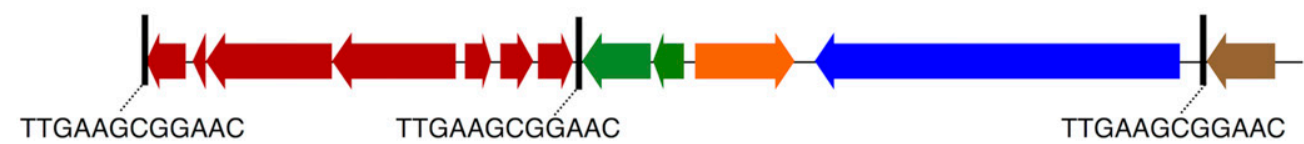

Fig. 5. Schematic genetic organization of toxicogenic regions (TRs) from different pathogenic Streptomyces isolates. Arrowed boxes represent the location and orientation of the open reading frames of thaxtomin A biosynthetic genes, integrase and recombination directionality factor (RDF), lantibiotic biosynthetic genes, conjugative genes, and aviX1. The sequences of attachment (att) sites at the junctions for TR1, TR2, and TR are also shown. 
we obtained three types of transconjugants: i) S. diastatochromogenes strains containing TR2 and the $S$. turgidiscabies PAI, resulting from the mobilization of TR2 from $S$. acidiscabies to $S$. diastatochromogenes, ii) $S$. diastatochromogenes strains containing TR1, TR2, and the $S$. turgidiscabies PAI, resulting from the mobilization of TR1 and TR2 together from $S$. acidiscabies to $S$. diastatochromogenes, and iii) $S$. acidiscabies strains containing TR1, TR2, and $S$. turgidiscabies PAI, resulting from the mobilization of $S$. turgidiscabies PAI from $S$. diastatochromogenes to $S$. acidiscabies. This mating experiment showed that, even though TR2 is absent in some pathogenic isolates, such as $S$. acidiscabies 84-104, these isolates can acquire TR2 from other isolates, leading to the site-specific accretion and cis-mobilization of resident TR1 and incoming TR2. In addition, these three types of transconjugants provide evidence for the exchange of the two currently known PAI types (S. turgidiscabies PAI and S. scabiei TR) between species.

\section{TR1 is fixed in pathogenic strains lacking TR2.}

We have demonstrated that S. scabiei TR1 is not mobile by itself and its transfer depends on TR2 (Zhang et al. 2016); however, comparative genomic analysis showed that many pathogenic isolates contain only TR1 but not TR2 (Fig. 5). We also observed the excision of $S$. acidiscabies TR1 in the absence of TR2 via repeat sequences different from S. scabiei (Fig. 4). It is possible that pathogenic strains that contain only TR1 use alternative mechanisms to disseminate TR1 to nonpathogenic species, which would be consistent with the observation that many pathogenic isolates only contain TR1. To test whether TR1 from those pathogenic strains that lack TR2 can be mobilized by alternative mechanisms, we carried out a third mating experiment, using $S$. acidiscabies $\triangle t x t A B$ with $S$. diastatochromogenes $\Delta n e c 1$ (Fig. 6C). Following selection with thiostrepton and kanamycin and confirmation with primers $a+b, k+1$, and $m+n$, we only obtained $S$. acidiscabies transconjugants containing TR1 and $S$. turgidiscabies PAI, which result from the mobilization of $S$. turgidiscabies PAI from $S$. diastatochromogenes to $S$. acidiscabies. Failure to detect the mobilization of $S$. acidiscabies TR1 to $S$. diastatochromogenes suggests that, without TR2, TR1 cannot be transferred. It therefore appears that strains containing TR1 but not TR2 are unlikely to result from the excision of TR1.

\section{DISCUSSION}

The capacity of bacteria to modulate their genome structure affects adaptation to changing environmental conditions and, therefore, impacts their evolution. The present study would appear to be the most comprehensive investigation of PAI diversity and evolution of pathogenicity in the genus Streptomyces, including 23 pathogenic isolates from nine known pathogenic species and a novel pathogenic species (Streptomyces sp. strain 96-12). We demonstrated that the entire TR region is a composite element that likely originated from the accretion of a putative ICE (TR2) and a CIME (TR1). Their site-specific accretion leads to the cis-mobilization and conjugative transfer of ThxA

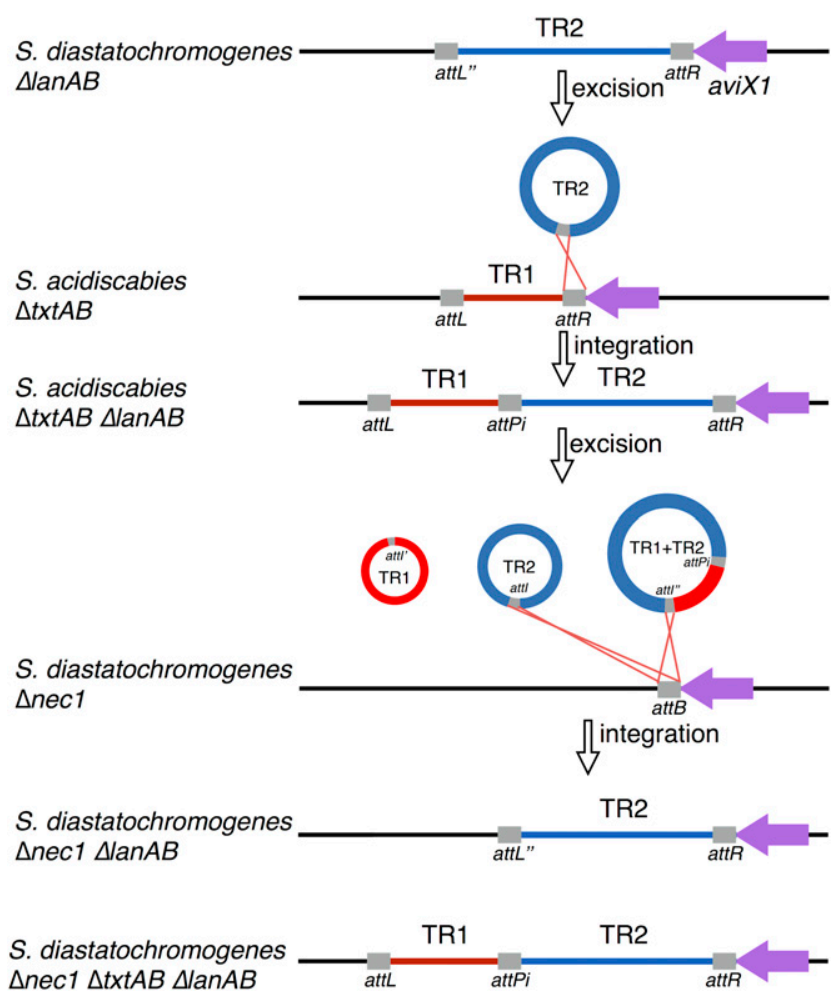

Fig. 7. Illustration of the site-specific accretion and cis-mobilization of TR1 and TR2. Streptomyces scabiei TR2 was site-specifically integrated into the attachment site at the $3^{\prime}$ end of the S. acidiscabies aviX1 gene. The composite toxicogenic region (TR) element can excise in three distinct forms: TR1, TR2, and the entire TR. TR2 alone or the whole TR can integrate into the $S$. diastatochromogenes chromosome. The cis-mobilization of TR1 and TR2 can lead to the emergence of novel pathogenic species.

\section{S. acidiscabies (TR1) S. diastatochromogenes (TR2) $\triangle t x t A B\left(\operatorname{kan}^{\mathrm{R}}\right) \quad \operatorname{Van} A B\left(\right.$ hyg $\left.^{\mathrm{R}}\right)$}

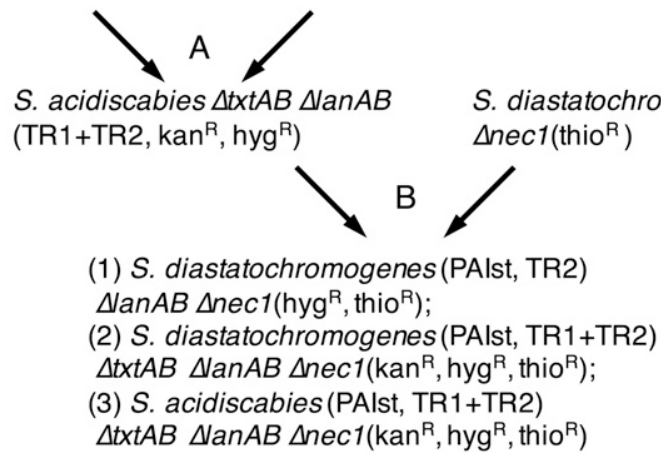

Fig. 6. Schematic representation of the mating experiments for investigation of the site-specific accretion and cis-mobilization of TR1 and TR2. A, The mating experiment between Streptomyces acidiscabies $\triangle t x A B$ and $S$. diastatochromogenes $\triangle$ lanAB, used to investigate site-specific accretion between TR1 and TR2. B, The mating experiment between S. acidiscabies $\triangle t x t A B \triangle l a n A B$ and $S$. diastatochromogenes $\Delta$ necl used to investigate the cis-mobilization of TR1 and TR2. C, The mating experiment between $S$. diastatochromogenes $\Delta n e c 1$ and $S$. acidiscabies $\triangle t x t A B$ used to investigate whether TR 1 can be transferred in strains lacking TR2. PAIst = pathogenicity island of $S$. turgidiscabies Car8. 
biosynthetic genes and can be responsible for the emergence of novel Streptomyces pathogenic species in agricultural systems. Since the Streptomyces genus is ubiquitous in soils, our study suggests that the spread of ThxA biosynthesis genes will continue to result in the emergence of new pathogenic Streptomyces species.

Recent sequencing efforts have provided more than 30 genomes of pathogenic Streptomyces isolates (Labeda 2016; Tomihama et al. 2016; Zhang et al. 2016). The comparative genomic analysis presented here allows us to characterize the pathogenicity factors in different pathogenic isolates. A striking finding is that PAI variation exists not only among different species but also among strains from the same species (Fig. 5). Seven $S$. acidiscabies isolates carried three different PAI structures, namely, TR1 alone (84-104, 85-06, 98-49, and FL01), the entire TR element (a10), and no TR (98-48 and NCPPB 4445). Interestingly, PAI structures of the three Japanese $S$. acidiscabies isolates (98-48, 98-49, and a10) differed from each other. Similarly, three $S$. niveiscabiei carried two different PAI structures. The isolate from South Korea (NRRL B-24457) carried the TR1 alone, but two isolates from Uruguay (ST1015 and ST1020) do not contain the TR element. If we sequence additional $S$. niveiscabiei genomes, we are likely to detect some isolates that carry the third TR version (the entire TR element). The variability in the structure of the TR element in newly emerged pathogenic species, such as S. acidiscabies and $S$. niveiscabiei, likely results from the instability of the composite TR element. Excision of an ICE could cause the loss of this ICE from the host; if ICE excision happens before chromosome replication or cell division, this element probably would not be passed down to progeny (Wozniak and Waldor 2010). Previous studies have shown that genomic islands can be lost from the bacterial population in the absence of selective pressure (Bellanger et al. 2011; Lechner et al. 2009). However, this leads to an important question regarding the lack of TR diversity in $S$. scabiei in the more than ten geographically distributed $S$. scabiei isolates that have been sequenced. It is possible that TR encodes functions that provide a significant selective advantage for $S$. scabiei.

Previously, the cis-mobilization of two CIMEs by related ICEs has been reported, i.e., the transfer of $\mathrm{CIMEL}_{3} \mathrm{CatR}_{3}$ by $\mathrm{ICESt}_{3}$ in Streptococcus thermophiles (Bellanger et al. 2011) and the transfer of CIME_Nem_tRNA ${ }^{L y s}$ by ICE_515_tRNA ${ }^{L y s}$ in Streptococcus agalactiae (Puymège et al. 2013). Our results indicate the cis-mobilization of TR1 by TR2 qualifies as another example of this mechanism. Additionally, studies of other genomic islands suggest that CIMEs probably stem from ICEs by deletion of genes involved in conjugation and integration (Pavlovic et al. 2004). It is possible that, in Streptomyces TR1, the CIME harboring the ThxA biosynthetic cluster has evolved by a similar mechanism. One hypothesis is that an ICE integrated into the $3^{\prime}$ end of aviXl could have lost its integration and conjugation genes (ICE1) while retaining two attachment sites (attL and attR), resulting in a CIME (TR1) (Fig. 8, step 1). A related ICE (ICE2/TR2) could then have been acquired by conjugation (Fig. 8, step 2) and integrated by site-specific recombination between its attI site and the attR site of TR1 (Fig. 8 , step 3), resulting in the composite structure attL-CIME (TR1)-attPi-ICE2 (TR2)-attR-aviX1. The whole structure could then have been excised by recombination among three attachment sites in three different forms (Fig. 8, step 4), i.e., as TR1, as TR2, or as the entire TR element. Then, TR2 or the entire TR could have been integrated into the attachment site at the $3^{\prime}$ end of the aviX1 gene of new hosts (Fig. 8, step 5). Acquisition of the entire TR element confers the pathogenic phenotype on new hosts, leading to the emergence of novel pathogenic species (Zhang et al. 2016). Due to the instability of the composite TR element, TR2 itself or the whole TR element may get lost and lead to diverse types of TR structures in the population of newly emerged pathogenic species (Fig. 8, step 6).

$S$. luridiscabiei, S. puniciscabiei, and S. niveiscabiei were reported to cause potato common scab in South Korea (Park et al. 2003a). Among these three species, only $S$. niveiscabiei produced thaxtomins (Park et al. 2003a). Interestingly, S. niveiscabiei strains that were isolated from pitted scab lesions in Uruguay in 2010 do not produce thaxtomins but are pathogenic (M. JuliaPianzzola personal communication). Comparative genomic analysis showed the isDDH values between $S$. niveiscabiei isolates from South Korea and Uruguay were only 87\%, suggesting that these isolates possibly are not derived from a common source. Indeed, although $S$. niveiscabiei has been discovered only in South Korea and Uruguay, this does not mean that other potatogrowing regions are free of this species. Our core genome-based phylogenetic analysis revealed the close relationship between $S$. niveiscabiei and $S$. acidiscabies. Owing to the poor characterization of this less-studied species, $S$. niveiscabiei isolates

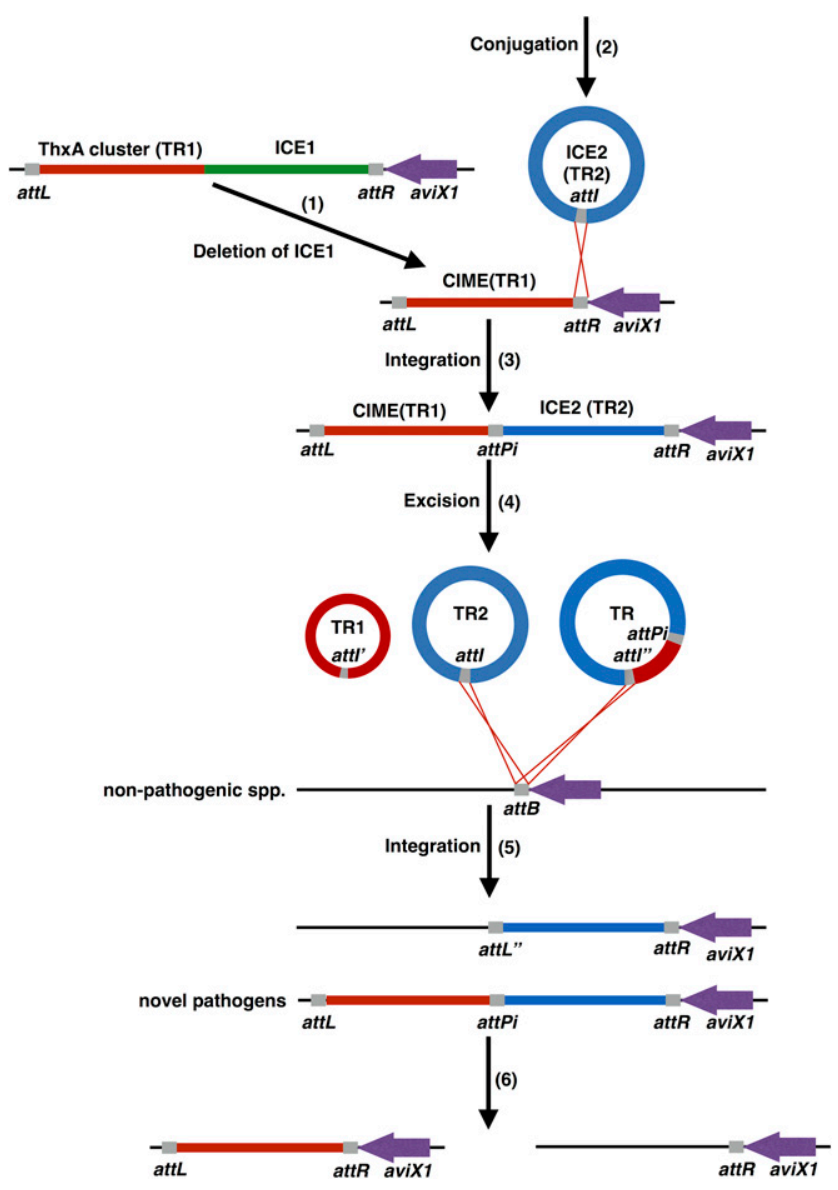

Fig. 8. Schematic representation of a hypothesis for the evolution of TR 1 and TR2 by deletion, site-specific accretion, and cis-mobilization. An integrative and conjugative element (ICE) integrated into the $3^{\prime}$ end of aviX1 could have lost its integration and conjugation genes while retaining two attachment (att) sites (attL and $a t t R)$, resulting in a cis-mobilizable element (CIME) (TR1) (step 1). In a subsequent event, a related ICE (TR2) could be acquired by conjugation (step 2) and site-specifically integrated between its attI site and the attR site of TR1 (step 3), resulting in the composite structure attL-CIME (TR1)-attPi-ICE2 (TR2)-attR-aviX1. The whole structure could be excised by recombination among three attachment sites in three different forms (step 4), i.e., as TR1, as TR2, or as the entire TR element. Then, TR2 or the entire TR could be integrated into the attachment site at the $3^{\prime}$ end of the aviX1 gene of new recipient strains (step 5). Due to the instability of the composite TR structure, TR2 or the whole TR could get lost (step 6), leading to the diversity of the TR structure within bacterial populations. 
could easily be misidentified as $S$. acidiscabies. $S$. niveiscabiei isolates from Uruguay lack the ThxA cluster, while isolates from South Korea harbor a ThxA cluster that is almost identical to the one in S. scabiei 87-22, despite large differences in their whole-genome sequences. These results suggest that, similar to $S$. acidiscabies, $S$. niveiscabiei is probably also an emergent pathogenic species and that the isolate from South Korea acquired the ThxA cluster via a recent HGT event.

Some $S$. turgidiscabies isolates from the potato fields of Finland infected by both $S$. scabiei and $S$. turgidiscabies were reported to contain a PAI with a structure similar to the TR element of S. scabiei 87-22 rather than to the PAI of $S$. turgidiscabies Car8, suggesting the exchange of PAI regions between two species (Aittamaa et al. 2010). Here, we demonstrated the exchange of the Car8-type PAI and TR-type PAI between S. acidiscabies and $S$. diastatochromogenes transconjugants. Such genetic exchange is critical to the evolution of pathogenicity of bacterial pathogens; the acquisition of new genes can enable pathogens to inhabit a new niche rapidly (Holden et al. 2009).

The mobility of the 674-kb PAI of $S$. turgidiscabies Car8 has been demonstrated (Kers et al. 2005), bringing up the possibility of the appearance of this large island in new pathogenic species. However, all newly identified pathogenic species contain the TR-type PAI or its derivative but not the S. turgidiscabies Car8-type PAI. It may be that the 674-kb Car8-type PAI cannot be stably transferred or maintained. This possibility is supported by an earlier report that considerable variability existed in the composition of PAI genes of S. turgidiscabies isolates in Finland (Aittamaa et al. 2010). Some isolates lack the fas operon, while other isolates lack all known virulence genes. However, in the present study, two Japanese $S$. turgidiscabies isolates (Car8 and T45) contained identical PAIs. These two isolates probably originated from the same source. Further genome sequencing projects should include additional $S$. turgidiscabies strains that contain diverse PAIs to investigate the evolution of mosaic regions of the Car8-type PAI.

Until recently, all Streptomyces species causing potato scab disease were thought to produce thaxtomins. Therefore, PCR primers that can amplify ThxA genes have been proposed for the detection of common scab pathogens (Flores-González et al. 2008; Lerat et al. 2009). However, a growing body of evidence suggests that other secondary metabolites may be pathogenicity factors. A Streptomyces isolate was reported to cause pitted scab lesions by producing an 18-membered macrolide named borrelidin (Cao et al. 2012). Recently, Fyans et al. (2016) isolated a Streptomyces strain from Newfoundland, Canada that has a severe pathogenic phenotype on several plant hosts. Symptoms were associated with an unidentified phytotoxin. S. reticuliscabiei, S. luridiscabiei, and S. puniciscabiei do not produce thaxtomins, but they are pathogenic species (Aittamaa et al. 2010; Park et al. 2003a). S. reticuliscabiei induces netted scab lesions on potato tubers (Aittamaa et al. 2010), while the latter two species cause typical potato scab lesions (Park et al. 2003a). Furthermore, two Uruguay $S$. niveiscabiei isolates (ST1015 and ST1020) that lack thaxtomin production cause deeppitted lesions on potato tubers (M. Julia-Pianzzola personal communication). Taken together, these results suggest a new paradigm for pathogenicity in Streptomyces. The secondary metabolite ThxA does appear to be the most common pathogenicity factor in Streptomyces species, but other secondary metabolites may serve this role in some plant-pathogenic strains.

\section{MATERIALS AND METHODS}

\section{Bacterial strains and culture conditions.}

Streptomyces strains were cultured at $28^{\circ} \mathrm{C}$ on International Streptomyces Project medium 4 (ISP4) agar medium or in tryptic soy broth (TSB) (BD Biosciences). All liquid cultures were shaken at $250 \mathrm{rpm}$. Genomic DNA was extracted from TSBgrown cultures using the MasterPure gram-positive DNA purification kit (Epicentre), based on the manufacturer's instructions.

\section{Virulence bioassays.}

To assess the virulence phenotype of different strains, an in vitro radish seedling bioassay was performed as described previously (Bignell et al. 2010b), except that 'German Giant' seeds (Burpee) were used in place of the 'Burpee White' variety. For each strain tested, mycelial suspensions were used to inoculate six newly germinated radish seedlings of similar size. The assay was performed three times, each of these experiments included three biological replicates.

\section{Analysis of ThxA production.}

Plant-pathogenic Streptomyces strains were cultured in TSB for 48 to $72 \mathrm{~h}$. Mycelia were then pelleted by centrifugation, were washed twice with sterile water, and were resuspended in sterile water, to obtain an optical density at $600 \mathrm{~nm}$ of 1.0. The mycelial suspension solution $(400 \mu \mathrm{l})$ was then inoculated into $3 \times 50 \mathrm{ml}$ of OBB medium (Johnson et al. 2007) in 250-ml flasks. After incubation for 6 days at $28^{\circ} \mathrm{C}$ with shaking $(250 \mathrm{rpm})$, ThxA was purified from the culture supernatants and was analyzed by highpressure liquid chromatography, as described previously (Johnson et al. 2007). All experiments were repeated at least three times using different biological replicates of the Streptomyces strains, with three technical repeats per strain. The pellets of the culture samples were dried and weighed as a measure of bacterial growth. The data for S. scabiei $87-22$ was normalized to $100 \%$. The data for all pathogenic isolates were square-root transformed to improve homogeneity of variance. Transformed data were subjected to one-way analysis of variance using SAS 9.4 (SAS Institute) and means were compared based on Tukey's honestly significantly different test at $P<0.05$.

\section{Genome sequencing and assembly.}

We sequenced the genomes of $S$. acidiscabies isolates using the Illumina-HiSeq 2500 paired-end technology. The SPAdes version 3.5.0 assembly algorithm (Bankevich et al. 2012) was used to perform genome assemblies. Prokka version 1.12-beta was used for gene-finding and annotation (Seemann 2014).

\section{Ortholog retrieval and single-copy cluster search.}

Streptacidiphilus albus JL83 and Kitasatospora setae NBRC 14216 were selected as outgroups to build the rooted phylogenetic tree. Protein sequences from 25 Streptomyces genomes and two outgroups were clustered into ortholog groups using OrthoMCL v1.4 (Li et al. 2003). OrthoMCL uses BLASTP similarity for clustering and was run using the default settings. Ortholog groups with a single copy per genome were selected as potential phylogenetic markers.

\section{Gene alignment and filtering criteria.}

To minimize the inclusion of alignments of low quality in the analysis, various filtering criteria were used as described previously (Zhang et al. 2016).

\section{Maximum likelihood phylogeny estimation.}

The gene alignment of nucleic acid sequences was used for maximum likelihood inference on the RAxML webserver (Stamatakis et al. 2008) with the GTR + G substation model, including an estimation of invariable sites. For inferring tree robustness, 1,000 bootstrap replicates were computed.

\section{ANI and isDDH estimation.}

The ANI analyses of whole-genome data were performed using the method proposed by Richter and Rosselló-Móra 
(2009). Pair-wise ANI values were obtained from nucmer (Delcher et al. 2002) with the script calculate_ani.py.

The isDDH was estimated using the genome-to-genome distance calculator (GGDC) (Auch et al. 2010b; Meier-Kolthoff et al. 2013). The contig files were uploaded to the GGDC 2.0 webserver, where isDDH calculations were performed using Formula 2 alone. We used the point estimates plus the $95 \%$ model-based confidence intervals as the isDDH estimates.

\section{Selection of transconjugant strains.}

Streptomyces strains were first grown on ISP4 plates, and spores were harvested after 5 to 7 days. Conjugal mating was carried out by mixing spores of donor and recipient strains and coculturing at $28^{\circ} \mathrm{C}$ on soya flour mannitol (SFM) plates (Kieser et al. 2000). After $48 \mathrm{~h}, 5 \mathrm{ml}$ of soft nutrient agar containing antibiotics was spread onto the plates to select transconjugants. Final concentrations of antibiotics were (per milliliter) $100 \mu \mathrm{g}$ of hygromycin $\mathrm{B}, 50 \mu \mathrm{g}$ of kanamycin, or $15 \mu \mathrm{g}$ of thiostrepton, singly or in combination. Qualitative PCR assays were used to confirm the transfer and integration of TR elements and PAI of S. turgidiscabies Car8.

\section{Excision assay.}

S. acidiscabies 84-104 was first streaked on ISP4 agar medium and was incubated at $28^{\circ} \mathrm{C}$ for 6 days. Three single colonies were picked and were transferred into TSB (BD Biosciences). All liquid cultures were shaken at $250 \mathrm{rpm}$ for 4 days. Genomic DNA was extracted from TSB-grown cultures using the MasterPure gram-positive DNA purification kit (Epicentre), based on the manufacturer's instructions. Qualitative nested PCR assayed were performed with $50 \mathrm{ng}$ genomic DNA, using primers TNi2 + TNi3 and TNi1 + TNi4 (Chapleau et al. 2016) for attPl and primers AcidF1 + AcidR1 and AcidF2 + AcidR2 for attB1. Each PCR reaction $(25 \mu \mathrm{l})$ was performed using $2 \times$ Apex Red Taq DNA polymerase master mix (Genesee Scientific) and primers at a final concentration of $0.4 \mu \mathrm{M}$. DNA amplification was performed for $4 \mathrm{~min}$ at $98^{\circ} \mathrm{C}$, followed by 30 cycles of $30 \mathrm{~s}$ at $98^{\circ} \mathrm{C}, 30 \mathrm{~s}$ at $57^{\circ} \mathrm{C}$, and $1 \mathrm{~min}$ at $72^{\circ} \mathrm{C}$, with a post-PCR extension at $72^{\circ} \mathrm{C}$ for $7 \mathrm{~min}$ and cooling to $4^{\circ} \mathrm{C}$. In the nested PCR, $0.5 \mu$ of PCR product from the firststep PCR was used as the template for the second-step PCR. All PCR products were also sequenced to confirm the excision.

\section{ACKNOWLEDGMENTS}

This work was partially supported by the Agriculture and Food Research Initiative Competitive Grants Program (grant 2010-65110-20416 from the United States Department of Agriculture National Institute of Food and Agriculture to R. Loria) and by University of Florida (UF) startup funds. Y. Zhang was the recipient of a UF alumni graduate fellowship.

\section{LITERATURE CITED}

Aittamaa, M., Somervuo, P., Laakso, I., Auvinen, P., and Valkonen, J. P. T. 2010. Microarray-based comparison of genetic differences between strains of Streptomyces turgidiscabies with focus on the pathogenicity island. Mol. Plant Pathol. 11:733-746.

Auch, A. F., Klenk, H.-P., and Göker, M. 2010a. Standard operating procedure for calculating genome-to-genome distances based on highscoring segment pairs. Stand. Genomic Sci. 2:142-148.

Auch, A. F., von Jan, M., Klenk, H.-P., and Göker, M. 2010b. Digital DNADNA hybridization for microbial species delineation by means of genome-to-genome sequence comparison. Stand. Genomic Sci. 2: $117-134$.

Bankevich, A., Nurk, S., Antipov, D., Gurevich, A. A., Dvorkin, M., Kulikov, A. S., Lesin, V. M., Nikolenko, S. I., Pham, S., Prjibelski, A. D., Pyshkin, A. V., Sirotkin, A. V., Vyahhi, N., Tesler, G., Alekseyev, M. A., and Pevzner, P. A. 2012. SPAdes: A new genome assembly algorithm and its applications to single-cell sequencing. J. Comput. Biol. 19: 455-477.
Bellanger, X., Morel, C., Gonot, F., Puymege, A., Decaris, B., and Guédon, G. 2011. Site-specific accretion of an integrative conjugative element together with a related genomic island leads to $\mathrm{cis}$ mobilization and gene capture. Mol. Microbiol. 81:912-925.

Bellanger, X., Payot, S., Leblond-Bourget, N., and Guédon, G. 2014. Conjugative and mobilizable genomic islands in bacteria: Evolution and diversity. FEMS Microbiol. Rev. 38:720-760.

Bignell, D. R. D., Huguet-Tapia, J. C., Joshi, M. V., Pettis, G. S., and Loria, R. 2010a. What does it take to be a plant pathogen: Genomic insights from Streptomyces species. Antonie van Leeuwenhoek 98:179-194.

Bignell, D. R. D., Seipke, R. F., Huguet-Tapia, J. C., Chambers, A. H., Parry, R. J., and Loria, R. 2010b. Streptomyces scabies 87-22 contains a coronafacic acid-like biosynthetic cluster that contributes to plantmicrobe interactions. Mol. Plant-Microbe Interact. 23:161-175.

Bouchek-Mechiche, K., Gardan, L., Normand, P., and Jouan, B. 2000. DNA relatedness among strains of Streptomyces pathogenic to potato in France: Description of three new species, S. europaeiscabiei sp. nov. and $S$. stelliscabiei sp. nov. associated with common scab, and $S$ reticuliscabiei sp. nov. associated with netted scab. Int. J. Syst. Evol. Microbiol. 50:91-99.

Bukhalid, R. A., Chung, S. Y., and Loria, R. 1998. nec1, a gene conferring a necrogenic phenotype, is conserved in plant-pathogenic Streptomyces spp. and linked to a transposase pseudogene. Mol. Plant-Microbe Interact. 11:960-967.

Bukhalid, R. A., Takeuchi, T., Labeda, D., and Loria, R. 2002. Horizontal transfer of the plant virulence gene, necl, and flanking sequences among genetically distinct Streptomyces strains in the Diastatochromogenes cluster. Appl. Environ. Microbiol. 68:738-744.

Burrus, V., and Waldor, M. K. 2004. Shaping bacterial genomes with integrative and conjugative elements. Res. Microbiol. 155:376-386.

Cao, Z., Khodakaramian, G., Arakawa, K., and Kinashi, H. 2012. Isolation of borrelidin as a phytotoxic compound from a potato pathogenic Streptomyces strain. Biosci. Biotechnol. Biochem. 76:353-357.

Chapleau, M., Guertin, J. F., Farrokhi, A., Lerat, S., Burrus, V., and Beaulieu, C. 2016. Identification of genetic and environmental factors stimulating excision from Streptomyces scabiei chromosome of the toxicogenic region responsible for pathogenicity. Mol. Plant Pathol. 17: 501-509.

de Klerk, A., McLeod, A., Faurie, R., and Van Wyk, P. S. 1997. Net blotch and necrotic warts caused by Streptomyces scabies on pods of peanut (Arachis hypogaea). Plant Dis. 81:958.

Delcher, A. L., Phillippy, A., Carlton, J., and Salzberg, S. L. 2002. Fast algorithms for large-scale genome alignment and comparison. Nucleic Acids Res. 30:2478-2483.

Fiedler, H.-P., Bruntner, C., Bull, A. T., Ward, A. C., Goodfellow, M., Potterat, O., Puder, C., and Mihm, G. 2005. Marine actinomycetes as a source of novel secondary metabolites. Antonie van Leeuwenhoek 87: $37-42$.

Flores-González, R. F., Velasco, I., and Montes, F. 2008. Detection and characterization of Streptomyces causing potato common scab in Western Europe. Plant Pathol. 57:162-169.

Fyans, J. K., Bown, L., and Bignell, D. R. D. 2016. Isolation and characterization of plant-pathogenic Streptomyces species associated with common scab-infected potato tubers in Newfoundland. Phytopathology 106:123-131

Goris, J., Konstantinidis, K. T., Klappenbach, J. A., Coenye, T., Vandamme, P., and Tiedje, J. M. 2007. DNA-DNA hybridization values and their relationship to whole-genome sequence similarities. Int. J. Syst. Evol. Microbiol. 57:81-91.

Goyer, C., Faucher, E., and Beaulieu, C. 1996. Streptomyces caviscabies sp. nov., from deep-pitted lesions in potatoes in Québec, Canada. Int. J. Syst. Evol. Microbiol. 46:635-639.

Healy, F. G., Wach, M., Krasnoff, S. B., Gibson, D. M., and Loria, R. 2000. The $t x t A B$ genes of the plant pathogen Streptomyces acidiscabies encode a peptide synthetase required for phytotoxin thaxtomin A production and pathogenicity. Mol. Microbiol. 38:794-804.

Holden, M. T. G., Heather, Z., Paillot, R., Steward, K. F., Webb, K., Ainslie, F., Jourdan, T., Bason, N. C., Holroyd, N. E., Mungall, K., Quail, M. A., Sanders, M., Simmonds, M., Willey, D., Brooks, K., Aanensen, D. M., Spratt, B. G., Jolley, K. A., Maiden, M. C. J., Kehoe, M., Chanter, N., Bentley, S. D., Robinson, C., Maskell, D. J., Parkhill, J., and Waller, A. S. 2009. Genomic evidence for the evolution of Streptococcus equi: Host restriction, increased virulence, and genetic exchange with human pathogens. PLoS Pathog. 5:e1000346.

Huguet-Tapia, J. C., Badger, J. H., Loria, R., and Pettis, G. S. 2011. Streptomyces turgidiscabies Car8 contains a modular pathogenicity island that shares virulence genes with other actinobacterial plant pathogens. Plasmid 65:118-124. 
Huguet-Tapia, J. C., Lefébure, T., Badger, J. H., Guan, D., Pettis, G. S., Stanhope, M. J., and Loria, R. 2016. Genome content and phylogenomics reveal both ancestral and lateral evolutionary pathways in plant pathogenic Streptomyces species. Appl. Environ. Microbiol. 82:2146-2155.

Huguet-Tapia, J. C., and Loria, R. 2012. Draft genome sequence of Streptomyces acidiscabies 84-104, an emergent plant pathogen. J. Bacteriol. 194:1847.

Johnson, E. G., Joshi, M. V., Gibson, D. M., and Loria, R. 2007. Cellooligosaccharides released from host plants induce pathogenicity in scab-causing Streptomyces species. Physiol. Mol. Plant Pathol. 71: 18-25.

Kers, J. A., Cameron, K. D., Joshi, M. V., Bukhalid, R. A., Morello, J. E., Wach, M. J., Gibson, D. M., and Loria, R. 2005. A large, mobile pathogenicity island confers plant pathogenicity on Streptomyces species. Mol. Microbiol. 55:1025-1033.

Kieser, T., Bibb, M. J., Buttner, M. J., Chater, K. F., and Hopwood, D. A. 2000. Practical Streptomyces Genetics. The John Innes Foundation, Norwich, U.K.

King, R. R., Lawrence, C. H., Calhoun, L. A., and Ristaino, J. B. 1994. Isolation and characterization of thaxtomin-type phytotoxins associated with Streptomyces ipomoeae. J. Agric. Food Chem. 42:1791-1794.

King, R. R., Lawrence, C. H., Clark, M. C., and Calhoun, L. A. 1989. Isolation and characterization of phytotoxins associated with Streptomyces scabies. J. Chem. Soc. Chem. Commun. 0:849-850.

Kritzman, G., Shani-Cahani, A., Kirshner, B., Riven, Y., Bar, Z., Katan, J., and Grinstein, A. 1996. Pod wart disease of peanuts. Phytoparasitica 24: 293-304.

Labeda, D. P. 2016. Taxonomic evaluation of putative Streptomyces scabiei strains held in the ARS culture collection (NRRL) using multi-locus sequence analysis. Antonie van Leeuwenhoek 109:349-356.

Lawrence, C. H., Clark, M. C., and King, R. R. 1990. Induction of common scab symptoms in aseptically cultured potato tubers by the vivotoxin, thaxtomin. Phytopathology 80:606-608.

Lechner, M., Schmitt, K., Bauer, S., Hot, D., Hubans, C., Levillain, E., Locht, C., Lemoine, Y., and Gross, R. 2009. Genomic island excisions in Bordetella petrii. BMC Microbiol. 9:141.

Lerat, S., Simao-Beaunoir, A.-M., and Beaulieu, C. 2009. Genetic and physiological determinants of Streptomyces scabies pathogenicity. Mol. Plant Pathol. 10:579-585.

Li, L., Stoeckert, C. J., Jr., and Roos, D. S. 2003. OrthoMCL: Identification of ortholog groups for eukaryotic genomes. Genome Res. 13:2178-2189.

Loria, R., Kers, J., and Joshi, M. 2006. Evolution of plant pathogenicity in Streptomyces. Annu. Rev. Phytopathol. 44:469-487.

Meier-Kolthoff, J. P., Auch, A. F., Klenk, H.-P., and Göker, M. 2013. Genome sequence-based species delimitation with confidence intervals and improved distance functions. BMC Bioinformatics 14:60.

Miyajima, K., Tanaka, F., Takeuchi, T., and Kuninaga, S. 1998. Streptomyces turgidiscabies sp. nov. Int. J. Syst. Bacteriol. 48:495-502.

Osborn, A. M., and Böltner, D. 2002. When phage, plasmids, and transposons collide: Genomic islands, and conjugative- and mobilizabletransposons as a mosaic continuum. Plasmid 48:202-212.

Park, D. H., Kim, J.-S., Kwon, S. W., Wilson, C., Yu, Y. M., Hur, J. H., and Lim, C. K. 2003a. Streptomyces luridiscabiei sp. nov., Streptomyces puniciscabiei sp. nov. and Streptomyces niveiscabiei sp. nov., which cause potato common scab disease in Korea. Int. J. Syst. Evol. Microbiol. 53:2049-2054.

Park, D. H., Yu, Y. M., Kim, J.-S., Cho, J. M., Hur, J. H., and Lim, C. K. 2003b. Characterization of Streptomycetes causing potato common scab in Korea. Plant Dis. 87:1290-1296.

Pavlovic, G., Burrus, V., Gintz, B., Decaris, B., and Guédon, G. 2004 Evolution of genomic islands by deletion and tandem accretion by sitespecific recombination: ICESt1-related elements from Streptococcus thermophilus. Microbiology 150:759-774.

Puymège, A., Bertin, S., Chuzeville, S., Guédon, G., and Payot, S. 2013. Conjugative transfer and cis-mobilization of a genomic island by an integrative and conjugative element of Streptococcus agalactiae. J. Bacteriol. 195:1142-1151.

Richter, M., and Rosselló-Móra, R. 2009. Shifting the genomic gold standard for the prokaryotic species definition. Proc. Natl. Acad. Sci. U.S.A. 106:19126-19131.

Salyers, A. A., Shoemaker, N. B., Stevens, A. M., and Li, L. Y. 1995 Conjugative transposons: An unusual and diverse set of integrated gene transfer elements. Microbiol. Rev. 59:579-590.

Seemann, T. 2014. Prokka: Rapid prokaryotic genome annotation. Bioinformatics 30:2068-2069.

Seipke, R. F., Kaltenpoth, M., and Hutchings, M. I. 2012. Streptomyces as symbionts: An emerging and widespread theme? FEMS Microbiol. Rev. 36:862-876.

Stamatakis, A., Hoover, P., and Rougemont, J. 2008. A rapid bootstrap algorithm for the RAxML Web servers. Syst. Biol. 57:758-771.

Takeuchi, T., Sawada, H., Tanaka, F., and Matsuda, I. 1996. Phylogenetic analysis of Streptomyces spp. causing potato scab based on 16S rRNA sequences. Int. J. Syst. Bacteriol. 46:476-479.

Tomihama, T., Nishi, Y., Sakai, M., Ikenaga, M., Okubo, T., and Ikeda, S. 2016. Draft genome sequences of Streptomyces scabiei S58, Streptomyces turgidiscabies T45, and Streptomyces acidiscabies a10, the pathogens of potato common scab, isolated in Japan. Genome Announc. 4:e00062-16

Wach, M. J., Krasnoff, S. B., Loria, R., and Gibson, D. M. 2007. Effect of carbohydrates on the production of thaxtomin A by Streptomyces acidiscabies. Arch. Microbiol. 188:81-88.

Wozniak, R. A. F., and Waldor, M. K. 2010. Integrative and conjugative elements: Mosaic mobile genetic elements enabling dynamic lateral gene flow. Nat. Rev. Microbiol. 8:552-563.

Zhang, Y., Bignell, D. R. D., Zuo, R., Fan, Q., Huguet-Tapia, J. C., Ding, Y., and Loria, R. 2016. Promiscuous pathogenicity islands and phylogeny of pathogenic Streptomyces spp. Mol. Plant-Microbe Interact. 29:640-650.

\section{AUTHOR-RECOMMENDED INTERNET RESOURCES}

Genome-to-Genome Distance Calculator (GGDC) 2.0 webserver: http://ggdc.dsmz.de/distcalc2.php

CIPRES Science Gateway RAxML webserver:

https://www.phylo.org/portal2/login!input.action

Widdowscript calculate_ani.py:

https:/github.com/widdowquinn/scripts/blob/master/bioinformatics/ calculate_ani.py 